\section{Proinflammatory Responses in the Murine Brain after Intranasal Delivery of Cholera Toxin: Implications for the Use of $\mathrm{AB}$ Toxins as Adjuvants in Intranasal Vaccines}

\author{
Michelle E. Armstrong, ${ }^{1}$ Ed C. Lavelle, ${ }^{1}$ Christine E. Loscher, ${ }^{1}$ \\ Marina A. Lynch, ${ }^{2}$ and Kingston H. G. Mills ${ }^{1}$
}

${ }^{1}$ Immune Regulation Research Group, School of Biochemistry and Immunology, and ${ }^{2}$ Department of Physiology and Trinity College Institute

of Neuroscience, Trinity College, Dublin, Ireland

Intranasal delivery of vaccines provides an attractive alternative to parenteral delivery, but it requires appropriate mucosal adjuvants. Cholera toxin (CT) is a powerful mucosal adjuvant, but it can undergo retrograde transport to the brain via the olfactory system after intranasal delivery. We demonstrate that intranasal delivery of CT increases the expression of interleukin- $1 \beta$, cyclooxygenase- 2 , and chemokine messenger RNA in the murine hypothalamus, whereas parenterally delivered CT has little effect. Our findings suggest that CT can induce proinflammatory mediators in the brain when it is administered intranasally but not parenterally, and they raise concerns about the use of $\mathrm{AB}$ toxins as adjuvants in intranasal vaccines.

Intranasally delivered vaccines provide an attractive alternative to parenteral vaccines, in that they facilitate the induction of mucosal immunity and eliminating the need for injection, but they require the inclusion of appropriate mucosal adjuvants. The AB-type toxins - cholera toxin (CT) and Escherichia coli heat-labile enterotoxin (LT)-have powerful mucosal adjuvant activity and enhance immune responses to antigens delivered orally, intranasally, and parenterally $[1,2]$. However, their in-

Received 1 February 2005; accepted 26 May 2005; electronically published 23 September 2005.

Potential conflicts of interest: K.H.G.M. is a cofounder, director, and shareholder in Opsona Therapeutics Ltd., a University Campus Company developing anti-inflammatory therapeutics. No other authors have competing financial interests.

Financial support: Health Research Board and Science Foundation Ireland.

Reprints or correspondence: Prof. Kingston H. G. Mills, Immune Regulation Research Group, School of Biochemistry and Immunology, Trinity College, Dublin 2, Ireland (kingston.mills @tcd.ie).

The Journal of Infectious Diseases 2005; 192:1628-33

(C) 2005 by the Infectious Diseases Society of America. All rights reserved. 0022-1899/2005/19209-0016\$15.00 herent toxicity, which is largely due to the ADP-ribosyltransferase activity of their enzymatic A subunit, hampers clinical use. Various nontoxic derivatives of CT and LT have been developed, many of which retain at least a portion of the adjuvant activity of the wild-type toxins [1]. However, concerns have been raised about the safety of AB-type toxins delivered intranasally [3].

The presence of a pentameric $\mathrm{B}$ subunit facilitates the binding of $\mathrm{AB}$ toxins to all nucleated cells, including neurons. It has been reported that intranasally delivered CT or the B subunit of CT can move, by retrograde transport, into the olfactory nerves/epithelium and olfactory bulb; this transport is facilitated by the ability of the B subunit to bind monosialoganglioside (GM-1) [3]. Furthermore, unlike the holototoxin, a non-GM-1-binding derivative of CT (CTA1-DD) did not bind to or accumulate in nervous tissues of the olfactory bulb [4]. In addition, cases of Bell palsy have been linked to an intranasally delivered influenza vaccine administered with LT as adjuvant, and the vaccine was subsequently withdrawn from clinical use [5]. These studies highlight the potential neurotoxicity of $\mathrm{AB}$ toxins after intranasal delivery and suggest that both the enzymatic and binding domains may contribute to adverse effects. However, to date, there have been no attempts to determine the functional significance of the accumulation of GM1-binding molecules in nervous tissue after intranasal delivery.

We have previously demonstrated that the parenteral administration of a whole-cell pertussis vaccine $(\mathrm{Pw})$ stimulated the production of interleukin (IL) $-1 \beta$ in the murine hypothalamus and hippocampus that was associated with fever and seizure responses, respectively [6, 7]. In addition, the parenteral injection of lipopolysaccharide (LPS), which is present at high concentrations in $\mathrm{Pw}_{\mathrm{w}}$, induces the expression of IL- $1 \beta$ in the brain, and this has been associated with febrile responses and cognitive dysfunction in rodents $[7,8]$. In the present study, we demonstrate that the intranasal, but not parenteral, administration of $\mathrm{CT}$ results in the induction of proinflammatory mediators (including IL-1 $\beta$ ) in the brain and that CT stimulates the production of IL- $1 \beta$ from mixed glial cells prepared from the brains of neonatal mice.

Materials and methods. Female BALB/c and C57BL/6 mice were purchased from Harlan. Mice were 8-10 weeks old at the initiation of experiments and were housed individually in ventilated cages. All experiments were performed in accordance with regulations of the Irish Department of Health, the European Union, and the Ethics Committee of Trinity College Dublin. CT, LPS $(10 \mu \mathrm{g})$, or PBS only was administered intranasally 
in 10-15 $\mu \mathrm{L}$ of PBS without the use of anesthetic or subcutaneously (sc) in $0.2 \mathrm{~mL}$ of PBS. Mice were killed by cervical dislocation 2 or $6 \mathrm{~h}$ later. Brains were rapidly removed, and hypothalamii and olfactory bulbs were dissected free. Individual hypothalamii, olfactory bulbs, and sections of liver were homogenized in $1 \mathrm{~mL}$ of TRI Reagent (Sigma) and frozen at $-80^{\circ} \mathrm{C}$ for subsequent mRNA analysis. Sections of liver were also homogenized in Tris- $\mathrm{HCl}$ buffer $(0.25 \mathrm{~mol} / \mathrm{L}[\mathrm{pH} 7.4])$ with $2 \%$ fetal calf serum for the estimation of IL- $1 \beta$ protein by ELISA (Duo-Set; R\&D Systems), as described elsewhere [7].

Primary cultures of mixed glia were prepared from whole brains of 0 -3-day-old BALB/c mice [8]. Cells were plated on poly-L-lysine-coated $(60 \mu \mathrm{g} / \mathrm{mL})$ coverslips in 24 -well plates at a density of 200,000 cells/well; these were fed every 3 days until confluence (day 10-14). Cells were then incubated with medium only, CT $(1 \mu \mathrm{g} / \mathrm{mL})$, or LPS $(100 \mathrm{ng} / \mathrm{mL})$ for $6 \mathrm{~h}$ at $37^{\circ} \mathrm{C}$ and then harvested for reverse-transcription polymerase chain reaction (PCR) or intracellular cAMP analysis by use of the Biotrak nonacetylation EIA system (Amersham Biosciences).

Total RNA was extracted from tissue samples and mixed glial cells by use of TRI Reagent, in accordance with the manufacturer's instructions. First-strand cDNA $(20 \mu \mathrm{L})$ was synthesized from $2 \mu \mathrm{g}$ of total RNA, as described elsewhere [9]. An aliquot $(2 \mu \mathrm{L})$ of each cDNA was used a template for PCR amplification with primers specific for IL-1 $\beta$ [9], cyclooxygenase (COX)-2 (forward, 5'-GTATCAGAACCGCATTGCCTCTGA-3'; reverse, 5'-CGGCTTCCAGTATTGAGGAGAACAGAT-3'), monocyte chemotactic protein (MCP)-1 (forward, 5'-TCTCTTCCTCCACCACCATGCAG-3'; reverse, 5'-GGAAAAATGGATCCACACCTTGC-3'), tumor necrosis factor (TNF) $-\alpha$ (forward, $5^{\prime}$-TGAACTTCGGGGTGATCGGTC-3'; reverse, 5'-AGCCTTGTCCCTTGAAGAGAAC- $3^{\prime}$ ), and macrophage inflammatory protein (MIP)-1 $\alpha$ (forward, $5^{\prime}$-CCCTTTTCTGTTCTGCTGACAAG-3'; reverse, $5^{\prime}$-GAAGAGTCCCTCGATGTGGCTA-3'), or $\beta$-actin [9], which was used as a housekeeping gene. Exon-spanning primers were used in all cases, to allow the discrimination of mRNA from contamination by genomic DNA. Amplified PCR products were separated by electrophoresis on $1.2 \%-2.0 \%$ agarose gels in the presence of ethidium bromide (10 $\mu \mathrm{L}$ of PCR reaction mix). The quantification of PCR products was performed by densitometric analysis of photographic negatives of agarose gels by use of GeneSnap acquisition and GeneTools analysis software (GeneGenius Gel Documentation and Analysis System; Syngene). For each mouse, the density of DNA amplicon bands were assessed, and values for mRNA are expressed in relative absorbance units. A band for a PBS control mouse was assigned an arbitrary value of $1 \mathrm{U}$, and the density of all other bands in that experiment was measured relative to that band. Results are expressed as ratios of the densitometer readings for the target genes divided by the reading for the $\beta$ actin gene.
One-way analysis of variance was used to test for statistical significance at each time point. Statistical significance was set at $P<.05$. Multiple comparisons between groups were assessed by use of the Tukey-Kramer post hoc test.

Results. We compared the effects of sc and intranasal administration of CT on proinflammatory gene expression in the hypothalamus of BALB/c mice. We had previously shown that parenterally delivered LPS can induce inflammatory cytokines in the hypothalamus and hippocampus of mice and rats and that this was associated with febrile responses and cognitive dysfunction [7, 8]. For the present study, we used LPS as a positive stimulator of inflammatory responses in the brain. We used the relatively high dose of $10 \mu \mathrm{g} /$ mouse to ensure a reproducible effect in a high proportion of mice. Preliminary experiments showed variable effects with lower doses. Intranasal administration was performed without anesthesia by use of a relatively low volume $(10-15 \mu \mathrm{L})$, to minimize transport into the respiratory tract. Compared with that in vehicle-treated control mice, a significant enhancement of the transcription of IL- $1 \beta$, TNF- $\alpha$, and MCP-1 was detected in the hypothalamus 2 and $6 \mathrm{~h}$ after the sc administration of LPS (figure 1A). Parenteral administration of LPS also enhanced the transcription of COX-2, MIP- $1 \alpha$, and MIP-2 in the hypothalamus at $2 \mathrm{~h}$ (figure 1 and data not shown). Parenteral administration of CT did not enhance mRNA expression of IL- $1 \beta$, TNF- $\alpha$, COX-2, or MCP-1 in the hypothalamus, compared with that in control mice, at either 2 or $6 \mathrm{~h}$ after treatment, but it did significantly enhance the expression of MIP- $1 \alpha$ at $2 \mathrm{~h}$. In contrast, the intranasal administration of CT significantly enhanced mRNA expression of IL- $1 \beta$, TNF- $\alpha$, COX-2, MIP- $1 \alpha$, and MCP-1 in the hypothalamus $6 \mathrm{~h}$ after treatment (figure 1). Furthermore, the intranasal delivery of CT increased mRNA expression of proinflammatory cytokines and chemokines in the hypothalamus of C57BL/6 mice (data not shown). Conversely, intranasally delivered LPS had no significant effect on cytokine mRNA expression in the hypothalamus (figure $1 B$ ).

It has been reported that CT can move into the olfactory bulb via retrograde transport along the olfactory nerves [3]. Here, we have demonstrated that the intranasal delivery of CT resulted in a significant increase in the mRNA expression of IL- $1 \beta$ in the olfactory bulb $6 \mathrm{~h}$, but not $2 \mathrm{~h}$, after treatment (figure $2 A$ ). LPS also significantly enhanced the transcription of IL- $1 \beta$ in the olfactory bulb $6 \mathrm{~h}$ after intranasal delivery. In contrast, intranasal delivery of CT had no effect on the mRNA expression of IL-1 $\beta$ (figure $2 B$ ) or the protein concentration of IL- $1 \beta$ (figure $2 D$ ) at a distant site, the liver. However, the sc administration of CT significantly enhanced the mRNA expression of IL-1 $\beta$ (figure $2 C$ ) and protein concentration (figure $2 D$ ) in the liver. Similarly, parenteral, but not intranasal, administration of LPS significantly increased the mRNA expression of IL- $1 \beta$ in the liver (figure $2 B$ and $2 C$ ). 
A
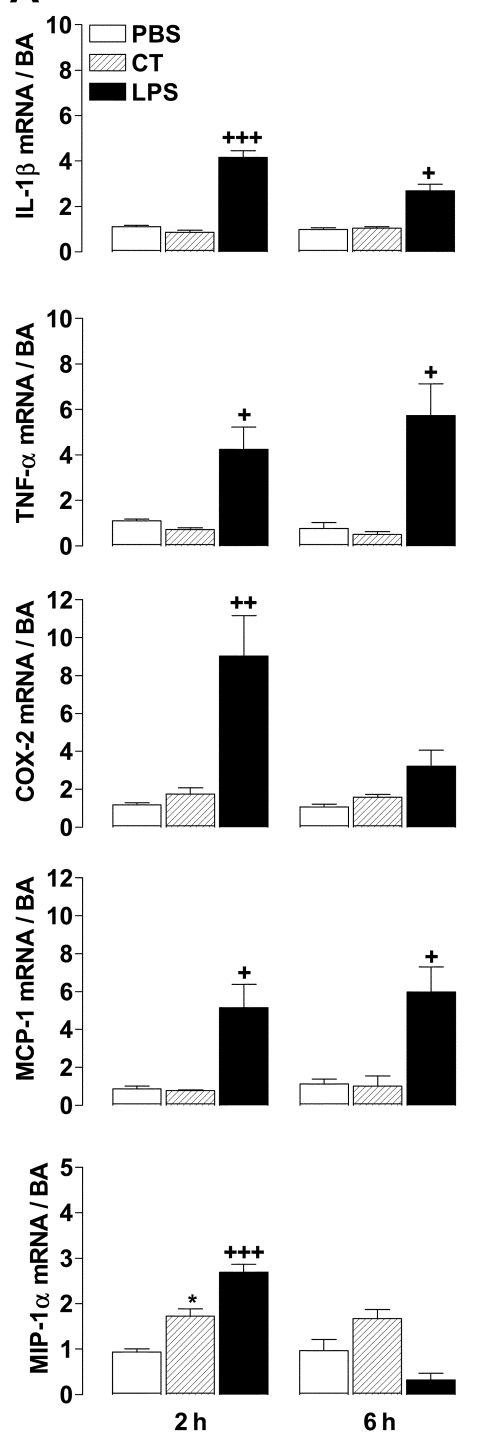

B
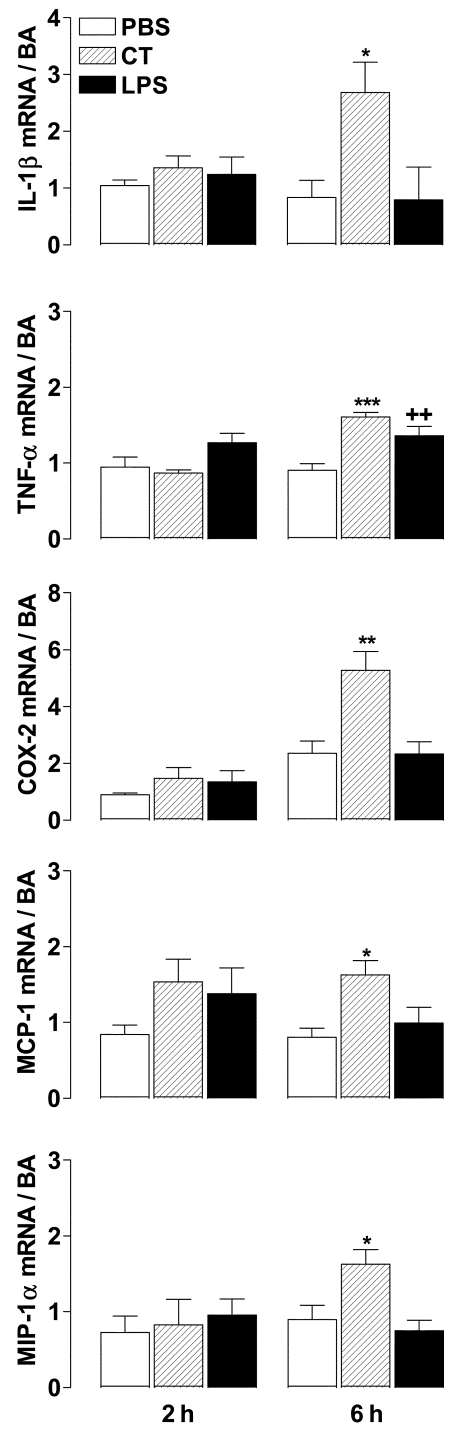

Figure 1. Enhancement of proinflammatory mediators by cholera toxin (CT) in the hypothalamus after intranasal but not parenteral delivery. CT, lipopolysaccharide (LPS; $10 \mu \mathrm{g}$ ), or PBS was administered to groups of $13 \mathrm{BALB} / \mathrm{c}$ mice subcutaneously (sc) (A) or intranasally (B). Mice were killed after 2 or $6 \mathrm{~h}$, and interleukin (IL)-1 $\beta$, tumor necrosis factor (TNF)- $\alpha$, cyclooxygenase (COX)-2, monocyte chemotactic protein (MCP)-1, and macrophage inflammatory protein (MIP)-1 $\alpha$ mRNA expression was determined by reverse-transcription polymerase chain reaction in the hypothalamus. Values for mRNA are given as the mean $\pm \operatorname{SE}(2 \mathrm{~h}, n=5 ; 6 \mathrm{~h}, n=8)$, are expressed in relative absorbance units, and are standardized per unit of $\beta$-actin (BA) per sample. ${ }^{+} P<.05,{ }^{+} P<.01$, and ${ }^{++} P<.001$, LPS- vs. PBS-treated mice; ${ }^{*} P<.05$ and ${ }^{* *} P<.01$, CT- vs. PBS-treated mice. Results are representative of 3 experiments.

One mechanism whereby LPS and CT may stimulate inflammatory cytokines in the brain is through the activation of astrocytes or microglial cells; therefore, we examined the ability of CT and LPS to stimulate inflammatory cytokines from mixed glial cells in culture. The stimulation of glial cells with CT resulted in a significant enhancement in intracellular cAMP concentrations, whereas LPS treatment had no effect (figure 2E). This is consistent with ADP ribosyltansferase activity of CT and its ability to elevate cAMP concentrations in other cell types [1]. In contrast, both CT and LPS enhanced the mRNA ex- pression of IL- $1 \beta$ and COX-2 (figure $2 F$ ) in mixed glial cells. $\mathrm{CT}$ also increased the mRNA expression of MIP-2 but not MCP-1, TNF- $\alpha$ or MIP- $1 \alpha$ at 6 h, whereas LPS increased the expression of all these mediators (data not shown). These findings suggest that CT and LPS have the capacity to stimulate proinflammatory cytokines in brain cells in vitro, and this may provide an explanation for the effects observed in vivo.

Discussion. The major finding of the present study is that the intranasal delivery of CT in mice is associated with significantly increased mRNA expression of proinflammatory 
A

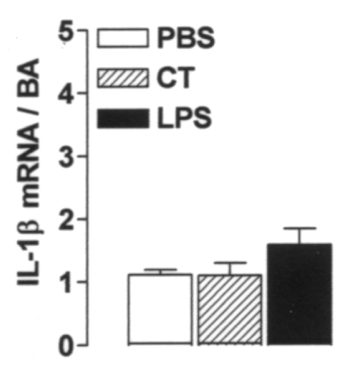

B

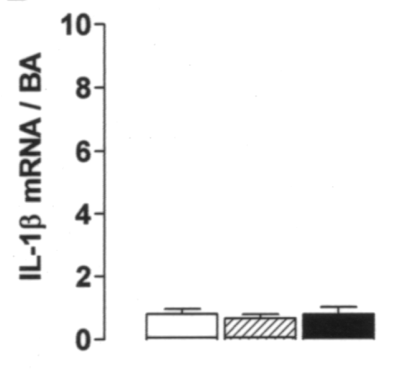

C

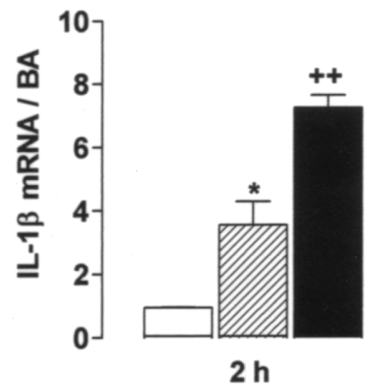

D

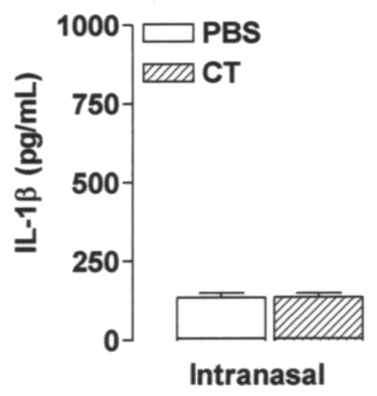

E

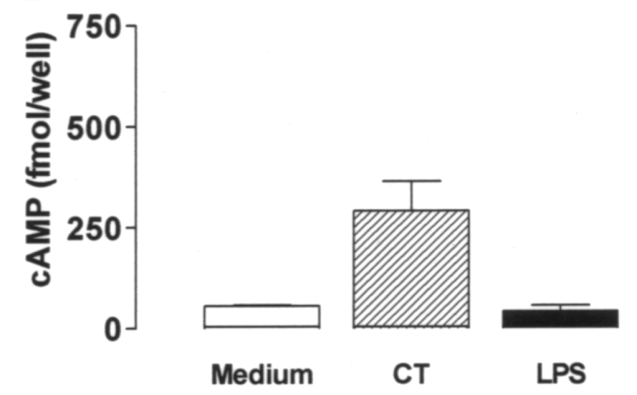

$\mathbf{F}$

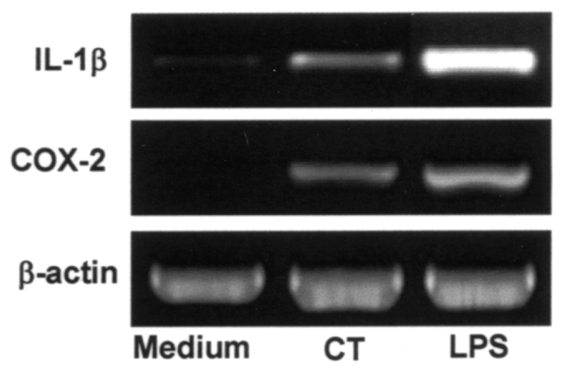

Figure 2. Induction of interleukin (IL)-1 $\beta$ expression in vivo by cholera toxin (CT) in the olfactory bulb and liver after intranasal and parenteral delivery, respectively, and in vitro from cultured glial cells. CT or lipopolysaccharide (LPS) was administered intranasally, and IL-1 $\beta$ mRNA expression was determined in the olfactory bulb $(A)$ and liver $(B)$ after 2 and $6 \mathrm{~h}$. CT and LPS were administered subcutaneously, and IL-1 $\beta$ mRNA expression was determined in the liver after 2 or $6 \mathrm{~h}(C)$. Values for mRNA are given as the mean \pm SE $(2 \mathrm{~h}, n=5 ; 6 \mathrm{~h}, n=8)$, are expressed in relative absorbance units, and are standardized per unit of $\beta$-actin (BA) per sample. IL-1 $\beta$ protein concentrations were determined by ELISA in liver samples recovered $6 \mathrm{~h}$ after subcutaneous or intranasal administration of CT (D) (subcutaneous, $n=6$; in, $n=8$ ). Mixed glial cells were stimulated with CT $(1 \mu \mathrm{g} / \mathrm{mL})$, LPS $(100 \mathrm{ng} / \mathrm{mL})$, or medium only, and intracellular cAMP concentration on cells was determined by EIA; results are given as the mean \pm SE for triplicate cultures (E) or for IL-1 $\beta$, cyclooxygenase (COX)-2, or BA mRNA expression in glial cells pooled from 4 wells determined by reversetranscription polymerase chain reaction (F) 6 h later. ${ }^{+} P<.05,{ }^{++} P<.01$, and ${ }^{+++} P<.001$, LPS- vs. PBS-treated mice; ${ }^{*} P<.05$ and ${ }^{* *} P<.01$, CT- vs. PBS-treated mice. Results are representative of 2 experiments.

mediators in the murine hypothalamus. Intranasally delivered vaccines can stimulate mucosal immunity, but they require powerful adjuvants such as AB-type toxins, which may be associated with a risk of neurological reactions due to transport to the brain via the olfactory system. It had previously been reported that GM-1-binding toxins undergo retrograde transport to the brain via the olfactory bulb [3]. In the present study, we observed significant increases in the mRNA expression of IL- $1 \beta$, COX-2, MCP-1, MIP- $1 \alpha$, and TNF- $\alpha$ in the hypothalamus of mice after the intranasal delivery of CT. Expression of these proinflammatory mediators were also enhanced in the murine hypothalamus after sc, but not intranasal, delivery of LPS. Febrile responses observed after the parenteral administration of LPS or Pw have been linked to an enhancement of IL- $1 \beta$, TNF- $\alpha$, COX-2, and MIP-1 expression in the hypothalamus $[6,10,11]$. Although the CT-induced responses are less profound and delayed, compared with those induced by parenterally delivered LPS, they were increased $>2$-fold and were significantly $(P<.05-.001)$ greater than those in control mice; this may have physiological consequences. Increases of $\leqslant 2$-fold in mRNA expression of IL- $1 \beta$ or COX-2 in the hypothalamus have been reported to be sufficient to induce fever 
[11] and cognitive dysfunction [8]. Increased expression of MCP-1 in the brain has been associated with enhanced bloodbrain barrier (BBB) permeability and has been implicated in the infiltration of mononuclear leukocytes into the central nervous system (CNS) in a number of neuroinflammatory conditions [12].

In contrast to intranasal delivery, the parenteral administration of CT had little effect on the mRNA expression of proinflammatory mediators in the hypothalamus. Intranasally delivered CT can gain access to the olfactory system through retrograde transport after binding to GM-1 on olfactory nerves [3]. Similarly, it has been shown that LT can move to the olfactory bulb within $6 \mathrm{~h}$ of intranasal delivery. This effect was reported to be dependent on the species and strain of experimental animals, with BALB/c mice being the most susceptible [13]. However, we observed proinflammatory effects with CT in BALB/c and C57BL/6 mice. The effects of CT in the hypothalamus after intranasal delivery may be due to its ability to move into the brain via retrograde transport along the olfactory nerve, thus bypassing the BBB. The parenteral administration of CT had no effect in the hypothalamus, which suggests that $\mathrm{CT}$ may be unable to cross the $\mathrm{BBB}$ or that it is sequestered by GM-1-expressing cells closer to the site of injection. In contrast, parenteral, but not intranasal, administration of LPS induced the production of proinflammatory mediators in the brain, which suggests that adjuvants that stimulate inflammatory responses through Toll-like receptors may be less reactogenic by the intranasal than by the parenteral route.

Our results do not rule out the possibility that peripheral signaling in the olfactory bulb might be capable of initiating intra-CNS synthesis of inflammatory mediators. However evidence from our own studies, as well as those of others [3, 4], suggests that CT may enter the brain and stimulate proinflammatory mediators from glial cells. Our data suggest that IL- $1 \beta$ induced in the hypothalamus after the intranasal delivery of CT is due to the local effects of CT in the brain. Intranasally administered CT enhanced the transcription of IL- $1 \beta$ in the olfactory bulb $6 \mathrm{~h}$ after treatment but had no effect on the production of IL- $1 \beta$ in the liver. This suggests that the intranasal delivery of CT has proximal effects in the olfactory bulb and hypothalamus but does not have effects at distal sites (such as the liver). In contrast, parenterally delivered CT increased the production of IL-1 $\beta$ in the liver. van Ginkel et al. [14] recently demonstrated that GM-binding pneumococci can use the olfactory nerves to target the brain and, thus, be detected in the brain in the absence of bacteremia.

The present study also demonstrates that CT can induce the transcription of IL- $1 \beta$ and COX-2 in a mixed glial-cell population of astrocytes and microglia. The activation of glial cells by CT was accompanied by an increase in intracellular cAMP levels, but that by LPS was independent of cAMP enhancement. The
ADP ribosyltransferase activity of the A subunit of CT activates adenylate cyclase, which leads to enhanced concentrations of intracellular cAMP, and this has been associated with the induction of cytokine secretion in dendritic cells and macrophages $[1,2]$. However, other immunomodulatory effects of CT and LT are independent of enzymatic activity. Nontoxic mutants of CT and LT retain certain of their adjuvant effects, and it has been argued that these may be safer for use in intranasal vaccines in humans [1]. Indeed, it has been demonstrated that an intranasally delivered nontoxic LT mutant, LTK63, did not induce inflammation-as detected by histological changes - in the olfactory bulb, brain, or meninges of outbred mice [15] and that it did not induce any serious adverse reactions as a component of an intranasal influenza vaccine tested in a clinical trial in humans (I. Stephenson, K. G. Nicholson, A. Rudin, A. Colegate, A. Podda, R. Bugarini, G. del Giudice, A. Minutello, S. Bonnington, J. Homgren, K.H.G.M., and M. C. Zambon, unpublished data). However, it has also been reported that the intranasal delivery of CT, LT, or the LT mutants LTR129G and LTS63K resulted in overt inflammation of the meninges and severe lesions in the olfactory bulb of BALB/c mice, whereas the B subunit of LT or a nonbinding mutant did not undergo retrograde transport to the olfactory bulb [16]. The present study in mice has demonstrated, to our knowledge, for the first time, that there may be a functional significance of the retrograde transport of CT into the CNS. The proinflammatory effects of intranasally delivered $\mathrm{CT}$ in the murine hypothalamus, along with its effect on cultured glial cells, suggests that the future clinical evaluation of LT and CT derivatives as adjuvants for intranasal vaccines should carefully monitor possible neurological effects in humans.

\section{References}

1. Pizza M, Giuliani MM, Fontana MG, et al. Mucosal vaccines: non toxic derivatives of LT and CT as mucosal adjuvants. Vaccine 2001; 19: 2534-41.

2. Lavelle EC, McNeela E, Armstrong ME, Leavy O, Higgins SC, Mills KHG. Cholera toxin promotes the induction of regulatory $\mathrm{T}$ cells specific for bystander antigens by modulating dendritic cell activation. J Immunol 2003; 171:2384-92.

3. van Ginkel FW, Jackson RJ, Yuki Y, McGhee JR. Cutting edge: the mucosal adjuvant cholera toxin redirects vaccine proteins into olfactory tissues. J Immunol 2000; 165:4778-82.

4. Eriksson AM, Schon KM, Lycke NY. The cholera toxin-derived CTA1DD vaccine adjuvant administered intranasally does not cause inflammation or accumulate in the nervous tissues. J Immunol 2004; 173 : 3310-19.

5. Mutsch M, Zhou W, Rhodes P, et al. Use of the inactivated intranasal influenza vaccine and the risk of Bell's palsy in Switzerland. N Engl J Med 2004; 350:896-903.

6. Loscher CE, Donnelly S, McBennett S, Lynch MA, Mills KHG. Proinflammatory cytokines in the adverse systemic and neurological effects associated with parenteral injection of a whole cell pertussis vaccine. Ann NY Acad Sci 1998; 856:274-7.

7. Donnelly S, Loscher CE, Lynch MA, Mills KHG. Whole-cell but not 
acellular pertussis vaccines induce convulsive activity in mice: evidence of a role for toxin-induced interleukin- $1 \beta$ in a new murine model for analysis of neuronal side effects of vaccination. Infect Immun 2001; 69:4217-23.

8. Nolan Y, Martin D, Campbell VA, Lynch MA. Evidence of a protective effect of phosphatidylserine-containing liposomes on lipopolysaccharide-induced impairment of long-term potentiation in the rat hippocampus. J Neuroimmunol 2004; 151:12-23.

9. Armstrong ME, Loscher CE, Lynch MA, Mills KHG. IL- $1 \beta$-dependent neurological effects of the whole cell pertussis vaccine: a role for IL1 -associated signalling components in vaccine reactogenicity. J Neuroimmunol 2003; 136:25-33.

10. Minano FJ, Fernandez-Alonso A, Myers RD, Sancibrian M. Hypothalamic interaction between macrophage inflammatory protein- 1 alpha (MIP-1 alpha) and MIP-1 beta in rats: a new level for fever control? J Physiol 1996; 491:209-17.

11. Fortier ME, Kent S, Ashdown H, Poole S, Boksa P, Luheshi GN. The viral mimic, polyinosinic:polycytidylic acid, induces fever in rats via an interleukin-1-dependent mechanism. Am J Physiol Regul Integr Comp Physiol 2004; 287:R759-66.

12. Huang D, Han Y, Rani MR, et al. Chemokines and chemokine receptors in inflammation of the nervous system: manifold roles and exquisite regulation. Immunol Rev 2000; 177:52-67.

13. Zurbriggen R, Metcalfe IC, Gluck R, Viret JF, Moser C. Nonclinical safety evaluation of Escherichia coli heat-labile toxin mucosal adjuvant as a component of a nasal influenza vaccine. Expert Rev Vaccines 2003; 2:295-304.

14. van Ginkel FW, McGhee JR, Watt JM, Campos-Torres A, Parish LA, Briles DE. Pneumococcal carriage results in ganglioside-mediated olfactory tissue infection. Proc Natl Acad Sci USA 2003; 100:14363-7.

15. Peppoloni S, Ruggiero P, Contorni M, et al. Mutants of the Escherichia coli heat-labile enterotoxin as safe and strong adjuvants for intranasal delivery of vaccines. Expert Rev Vaccines 2003; 2:285-93.

16. National Institute of Allergy and Infectious Diseases. Safety evaluation of toxin adjuvants delivered intranasally. Available at: http://www.niaid .nih.gov/dmid/enteric/intranasal.htm. Accessed 19 September 2005. 